\title{
Rapid antigen testing and non-infectious shedding of SARS-Cov2
}

\author{
Günter Weiss ${ }^{1}\left[\right.$ [ Rosa Bellmann-Weiler ${ }^{1}$
}

Received: 11 December 2020 / Accepted: 16 December 2020 / Published online: 2 January 2021

(c) Springer-Verlag GmbH, DE part of Springer Nature 2021

We read with interest and appreciated the comment of Raveh et al. (INFE-D-20-02430) regarding our recent publication describing the clinical potential of a rapid antigen test to identify SARS-CoV2 infection in hospitalized COVID-19 patients with symptoms and RT-PCR confirmed diagnosis [1]. Therein, we found that the Panbio ${ }^{\mathrm{R}}$ Antigen test had a good sensitivity in patients with high viral loads $(\mathrm{Ct}$ values $<25$ ) with almost $86 \%$ of subjects being correctly detected. We also found that the test remained positive in a relevant number of patients, which according to the classification of the German Robert Koch Institute (RKI) or the US Center of disease control (CDC) would be considered as non-infectious.

In contrast to the argumentation of Raveh et al., our primary intension was to verify the clinical utility of this test to identify infected subjects as sensitivity and specificity data given by the producers are often lower in the clinical setting. As we only tested patients with positive PCR results we could not provide any information on the specificity of the tests as we clearly stated in our manuscript. We also did not have the intention to study the function of the test as a rule out strategy to differentiate potentially infectious from non-infectious subjects on the basis of high $\mathrm{Ct}$ values used as a gold standard. We fully agree with the authors that $\mathrm{Ct}$ values may largely vary according to the test method used and that results may even differ between various laboratories using the same tests [2]. Thus, when using $\mathrm{Ct}$ values for interpretation of possible infectivity at least two factors are really important. First, to use the same method, apparatus and laboratory for PCR analysis to avoid inter- and intraassay variances and second, to interpret the results always in the context of the clinical presentation of the patients including timing of onset and severity of symptoms as well

Günter Weiss

Guenter.weiss@i-med.ac.at

1 Department of Internal Medicine II, Infectious Diseases, Immuology, Rheumatology, Pneumology, Medical University of Innsbruck, Anichstr. 35, 6020 Insnbruck, Austria as the duration and course of the disease or also related to co-morbidities or immune deficiencies which could result in reduced viral clearance or prolonged viral shedding by the host.

As this was a pilot study, we did not show data on the timing of antigen test according to symptom onset of patients. However, patients during the first days of hospitalization had the lowest $\mathrm{Ct}$ values and those patients were mostly tested positive by the antigen test. Nonetheless, we agree with Raveh et al. that we had a selected group of patients, namely those who needed hospitalization due to severe disease. As hospitalization occurred mostly several days after symptom onset [3], we cannot exactly predict whether the sensitivity of the antigen test would be in a comparable range if test samples would have been taken immediately after onset of symptoms. This is a further need for future studies as well as the evaluation of the specificity and sensitivity of antigen tests for public screening along with a professional communication of the advantages and limitations of such strategies. Finally, we also await the clinical evaluation and follow up of symptomatic or asymptomatic patients who were tested positive with the antigen test but negative with PCR during such screenings, as the PCR also has limitations in sensitivity due to multiple factors discussed elsewhere $[4,5]$.

\section{References}

1. Lanser L, Bellmann-Weiler R, Ottl KW, Huber L, Griesmacher A, Theurl I, Weiss G. Evaluating the clinical utility and sensitivity of SARS-CoV-2 antigen testing in relation to RT-PCR Ct values. Infection. 2020. https://doi.org/10.1007/s15010-020-01542-0.

2. Garg A, Ghoshal U, Patel SS, Singh DV, Arya AK, Vasanth S, Pandey A, Srivastava N. Evaluation of seven commercial RT-PCR kits for COVID-19 testing in pooled clinical specimens. J Med Virol. 2020. https://doi.org/10.1002/jmv.26691.

3. Bellmann-Weiler R, Lanser L, Barket R, Rangger L, Schapfl A, Schaber M, Fritsche G, Woll E, Weiss G. Prevalence and predictive value of anemia and dysregulated iron homeostasis in patients with COVID-19 infection. J Clin Med. 2020;9:2429.

4. Mustafa Hellou M, Gorska A, Mazzaferri F, Cremonini E, Gentilotti E, De Nardo P, Poran I, Leeflang MM, Tacconelli E, Paul 
M. Nucleic acid amplification tests on respiratory samples for the diagnosis of coronavirus infections: a systematic review and metaanalysis. Clin Microbiol Infect. 2020. https://doi.org/10.1016/j. cmi.2020.11.002.
5. Bouiller K, Humbert S, Payet-Revest C, Brunel AS, Mareshal A, Lepiller Q, Grillet F, Chirouze C. Utility of CT scan in patients with initial negative PCR for SARS-CoV2: a report of three cases. Infection. 2020;48:945-8. 